\title{
ON CONDITIONS PROVIDED BY NILRADICALS
}

\author{
Hong Kee Kim, Nam Kyun Kim, Mun Seob Jeong, Yang Lee, \\ Sung Ju Ryu, and Dong Eun Yeo
}

\begin{abstract}
A ring $R$ is called $I F P$, due to Bell, if $a b=0$ implies $a R b=0$ for $a, b \in R$. Huh et al. showed that the IFP condition is not preserved by polynomial ring extensions. In this note we concentrate on a generalized condition of the IFPness that can be lifted up to polynomial rings, introducing the concept of quasi-IFP rings. The structure of quasi-IFP rings will be studied, characterizing quasi-IFP rings via minimal strongly prime ideals. The connections between quasi-IFP rings and related concepts are also observed in various situations, constructing necessary examples in the process. The structure of minimal noncommutative (quasi-)IFP rings is also observed.
\end{abstract}

\section{Quasi-IFP rings and related concepts}

Throughout every ring is associative with identity unless otherwise stated.

Given a ring $R, J(R), N_{*}(R), N^{*}(R)$, and $N(R)$ denote the Jacobson radical, the prime radical, the upper nilradical (i.e., sum of nil ideals), and the set of all nilpotent elements in $R$, respectively. Note $N_{*}(R) \subseteq N^{*}(R) \subseteq N(R)$. Based on Artin and Wedderburn, the Wedderburn radical of a $\operatorname{ring} R$ means the sum of all nilpotent ideals in $R$ (in spite of this sum being not a radical, it was given the name), written by $N_{0}(R) . X$ denotes a nonempty set of commuting indeterminates over rings. Let $R$ be a ring. The polynomial ring over $R$ with $X$ is denoted by $R[X]$, and if $X$ is a singleton, say $X=\{x\}$, then we write $R[x]$ in place of $R[\{x\}]$. The $n$ by $n$ matrix ring over a $\operatorname{ring} R$ is denoted by $\operatorname{Mat}_{n}(R)$, and $e_{i j}$ denotes the $n$ by $n$ matrix with $(i, j)$-entry 1 and zero elsewhere.

Received November 29, 2007.

2000 Mathematics Subject Classification. 16D25, 16N40, 16 S36.

Key words and phrases. IFP ring, quasi-IFP ring, Wedderburn radical, nilradical, polynomial ring.

The fifth named author was financially supported by Pusan National University in program Post-Doc. 2008. The first named author was supported by the fund of Research Promotion Program(RPP-2007-000), Gyeongsang National University. The second named author was supported by a grant from Hanbat National University Academy in 2008. The fourth named author was supported by the Korea Research Foundation Grant funded by the Korean Government(MOEHRD)(KRF-2005-015-C00011). 
$r_{R}(-)$ (resp. $\ell_{R}(-)$ ) is used for the right (resp. left) annihilator over a ring $R$, i.e., $r_{R}(S)=\{a \in R \mid s a=0$ for all $s \in S\}$ (resp. $\ell_{R}(S)=\{b \in R \mid b s=$ 0 for all $s \in S\}$ ), where $S \subseteq R$ or $S$ is a subset of a right (resp. left) $R$-module. Write $r_{R}(a)$ (resp. $\ell_{R}(a)$ ) in place of $r_{R}(\{a\})$ (resp. $\left.\ell_{R}(\{a\})\right) . a \in R$ is said to be right (resp. left) regular if $r_{R}(a)=0$ (resp. $\left.\ell_{R}(a)=0\right)$. A regular element is defined to be both left and right regular. $a \in R$ is called a left (resp. right) zero-divisor if $r_{R}(a) \neq 0$ (resp. $\ell_{R}(a) \neq 0$ ). A zero-divisor means an element that is neither right nor left regular. A domain means a ring whose nonzero elements are regular.

A prime ideal $P$ of a ring $R$ is called completely prime if $R / P$ is a domain. According to Kim et al. [17], a ring is called nil-semisimple if it has no nonzero nil ideals. Nil-semisimple rings are clearly semiprime, but semiprime rings need not be nil-semisimple as can be seen by [13, Example 1.2 and Proposition 1.3]. Due to Rowen [22, Definition 2.6.5], an ideal $P$ of a ring $R$ is called strongly prime if $P$ is prime and $R / P$ is nil-semisimple. Maximal ideals and completely prime ideals are clearly strongly prime. Nil-semisimple rings need not be prime as can be seen by direct products of reduced rings; and prime rings also need not be nil-semisimple as can be seen by [13, Example 1.2 and Proposition 1.3]. Note that any strongly prime ideal contains a minimal strongly prime ideal. $N^{*}(R)$ of a ring $R$ is the unique maximal nil ideal of $R$ by [22, Proposition 2.6.2], and with the help of [22, Proposition 2.6.7] we have

$$
\begin{aligned}
N^{*}(R) & =\{a \in R \mid R a R \text { is a nil ideal of } R\} \\
& =\bigcap\{P \mid P \text { is a (minimal) strongly prime ideal of } R\} .
\end{aligned}
$$

A ring $R$ is called reduced if $N(R)=0$. Due to Marks [20], a ring $R$ is called $N I$ if $N^{*}(R)=N(R)$. Reduced rings are clearly NI. Note that $R$ is NI if and only if $N(R)$ forms an ideal if and only if $R / N^{*}(R)$ is reduced. Hong et al. [9, Corollary 13] showed that a ring $R$ is NI if and only if every minimal strongly prime ideal of $R$ is completely prime. According to Birkenmeier et al. [3], a ring $R$ is called 2-primal when $N_{*}(R)=N(R)$. It is obvious that a ring $R$ is 2-primal if and only if $R / N_{*}(R)$ is reduced. Birkenmeier et al. [3, Proposition 2.6] proved that a ring $R$ is 2-primal if and only if so is $R[X]$. Shin showed that a ring $R$ is 2-primal if and only if every minimal prime ideal of $R$ is completely prime [23, Proposition 1.11]. 2-primal rings are clearly NI, but the converse need not hold by Hwang et al. [13, Example 1.2] or Marks [20, Example 2.2].

There are various conditions between the commutativity and the NIness. Among them we concentrate on the insertion-of-factors-property (simply IFP) and the 2-primalness. Due to Bell [2], a right (or left) ideal $I$ of a $\operatorname{ring} R$ is said to have the IFP if $a b \in I$ implies $a R b \subseteq I$ for $a, b \in R$. So a $\operatorname{ring} R$ is called IFP if the zero ideal of $R$ has the IFP. Shin [23] used the term $S I$ for the IFP; while IFP rings are also known as semicommutative in Narbonne's paper 
[21]. Reduced rings are IFP by a simple computation. IFP rings are 2-primal by [23, Theorem 1.5].

A ring is called Abelian if each idempotent is central. IFP rings are Abelian by a simple computation, but quasi-IFP rings need not be Abelian as can be seen by 2 by 2 upper triangular matrix rings over reduced rings.

In this note a ring $R$ will be called quasi-IFP provided that $\sum_{i=0}^{n} R a_{i} R$ is nilpotent whenever $\sum_{i=0}^{n} a_{i} x^{i} \in R[x]$ is nilpotent. As we see below, IFP rings are quasi-IFP and this implication is irreversible.

Lemma 1.1. (1) $A$ ring $R$ is IFP if and only if $r_{R}(S)$ is an ideal of $R$ for any $S \subseteq R$ if and only if $\ell_{R}(S)$ is an ideal of $R$ for any $S \subseteq R$.

(2) IFP rings are quasi-IFP.

(3) Quasi-IFP rings are 2-primal.

(4) Subrings of quasi-IFP rings are quasi-IFP.

Proof. (1) and (2) are proved by [23, Lemma 1.2] and [8, Lemma 1.1(4)] respectively.

(3) Let $R$ be a quasi-IFP ring and $0 \neq a \in N(R)$. Then $R a R$ is nilpotent and so $R a R \subseteq N_{*}(R)$, getting $N_{*}(R)=N(R)$.

(4) Let $R$ be a quasi-IFP ring and $S$ be a subring of $R$. Let $\sum_{i=0}^{n} a_{i} x^{i} \in$ $N(S[x])$, then $\sum_{i=0}^{n} a_{i} x^{i} \in N(R[x])$. Since $R$ is quasi-IFP, $\sum_{i=0}^{n} R a_{i} R$ is nilpotent. Immediately $\sum_{i=0}^{n} S a_{i} S$ is nilpotent.

The implications in Lemma 1.1(2),(3) are irreversible by the following. Given a ring $R$, the $n$ by $n$ upper triangular matrix ring over $R$ is denoted by $U_{n}(R)$.

Example 1.2. (1) There exists a quasi-IFP ring but not IFP. Let $R=U_{n}(S)$ over a reduced ring $S$ with $n \geq 2$. Note $R[X] \cong U_{n}(S[X])$. Note

$$
N_{*}(R[X])=\{A \in R \mid \text { the diagonal of } A \text { is zero }\}[X]=N_{0}(R[X]) .
$$

So we get a reduced factor $\operatorname{ring} \frac{R[X]}{N_{*}(R[X])} \cong \oplus_{i=1}^{n} T_{i}$ with $T_{i}=S[X]$ for all $i$, entailing $N_{*}(R[X])=N(R[X])$. Hence $\sum_{i=0}^{m} R a_{i} R$ is nilpotent (actually $\left.\left(\sum_{i=0}^{m} R a_{i} R\right)^{n}=0\right)$ whenever $\sum_{i=0}^{m} a_{i} x^{i} \in N(R[x])$. Thus $R$ is quasi-IFP but $R$ is not IFP since $R$ is non-Abelian.

(2) There exists a 2-primal ring but not quasi-IFP. Let $K$ be a field and $K\{x, y\}$ be the free algebra generated by noncommuting indeterminates $x, y$ over $K$. The following construction is essentially due to [17, Example 2.4(2)]. Consider an infinite word

$$
w=y x y x x y x x y x x x y x x x x \cdots=\prod_{i=1}^{\infty} y x^{i} .
$$

Let $I$ be the ideal of $K\{x, y\}$ generated by the set of all words each of which is not a subword of $w$, and define $R=K\{x, y\} / I . y+I$ is nilpotent, however $R(y+I) R$ is non-nilpotent by the computation in [17, Example 2.4(2)]. Thus $R$ is not quasi-IFP, but 2-primal by the argument in [17, Example 2.4(2)]. 
In the following lemma we find various condition equivalent to the quasiIFPness. Lambek [11] called a ring $R$ symmetric when $r$ st $=0$ implies $r t s=0$ for all $r, s, t \in R$, proving that a ring $R$ is symmetric if and only if $r_{1} r_{2} \cdots r_{n}=$ 0 , with $n$ any positive integer, implies $r_{\sigma(1)} r_{\sigma(2)} \cdots r_{\sigma(n)}=0$ for any permutation $\sigma$ of the set $\{1,2, \ldots, n\}$ and $r_{i} \in R[18$, Proposition 1]. Symmetric rings are IFP obviously, and reduced rings are symmetric by $[18$, Section $1(\mathrm{G})]$.

Lemma 1.3. For a ring $R$ the following conditions are equivalent:

(1) $R$ is quasi-IFP;

(2) $\sum_{i=0}^{n} R a_{i} R$ is nilpotent whenever $a_{0}+\sum_{i=1}^{n} a_{i} P_{i} \in R[X]$ is nilpotent, where each $P_{i}$ is a finite product of indeterminates in $X$;

(3) $N(R)=N_{0}(R)$ (i.e., $R a R$ is nilpotent for any $a \in N(R)$ );

(4) $R$ is 2-primal with $N_{0}(R)=N_{*}(R)$;

(5) $R$ is NI with $N_{0}(R)=N^{*}(R)$;

(6) Every minimal prime ideal of $R$ is completely prime with

$$
N_{0}(R)=N_{*}(R)
$$

(7) Every minimal strongly prime ideal of $R$ is completely prime with

$$
N_{0}(R)=N^{*}(R)
$$

(8) $R / N_{0}(R)$ is a subdirect product of domains;

(9) $R / N_{0}(R)$ is a reduced ring;

(10) $R / N_{0}(R)$ is a symmetric ring with $N_{0}(R)=N_{*}(R)$.

Proof. $(1) \Rightarrow(3),(3) \Rightarrow(4),(4) \Rightarrow(5),(5) \Rightarrow(3),(7) \Rightarrow(8),(8) \Rightarrow(9),(9) \Rightarrow(3)$, and $(2) \Rightarrow(1)$ are obvious. $(4) \Leftrightarrow(6)$ and $(5) \Leftrightarrow(7)$ are obtained from [23, Proposition 1.11] and [9, Corollary 13] respectively.

$(1) \Rightarrow(2)$ : Let $R$ be quasi-IFP and $a_{0}+\sum_{i=1}^{n} a_{i} P_{i} \in N(R[X])$. Since $R$ is 2-primal by Lemma $1.1(3), \frac{R[X]}{N_{*}(R[X])} \cong \frac{R}{N_{*}(R)}[X]$ is reduced and so $N(R[X]) \subseteq$ $N_{*}(R[X])=N_{*}(R)[X]$, concluding that each $a_{i}$ is in $N_{*}(R)$ for $i=0, \ldots, n$. Since $R$ is quasi-IFP, each $R a_{i} R$ is nilpotent and thus $\sum_{i=0}^{n} R a_{i} R$ is nilpotent.

$(3) \Rightarrow(1)$ : Let $N(R)=N_{0}(R)$ and $\sum_{i=0}^{n} a_{i} x^{i} \in N(R[x])$. Then $N_{*}(R)=$ $N_{0}(R)$ and $\frac{R[x]}{N_{*}(R[x])} \cong \frac{R}{N_{*}(R)}[x]$ is reduced, concluding that $N(R[x]) \subseteq N_{*}(R[x])$ $=N_{*}(R)[x]=N_{0}(R)[x]$. Thus each $a_{i}$ is in $N_{0}(R)$ for $i=0, \ldots, n$; hence each $R a_{i} R$ is nilpotent, entailing $\sum_{i=0}^{n} R a_{i} R$ is nilpotent.

Reduced rings are symmetric by [18], obtaining $(9) \Rightarrow(10)$. Symmetric rings are 2-primal, and so $R / N_{0}(R)$ is reduced when $R / N_{0}(R)$ is symmetric with $N_{0}(R)=N_{*}(R)$. Thus $(10) \Rightarrow(9)$ holds.

By Lemma 1.3(3), the quasi-IFPness is equal to the $W_{1}$-reducedness in [17]. The subcondition " $N_{0}(R)=N_{*}(R)$ " in the condition (10) in Lemma 1.3 is not superfluous by the following. Let $R$ be an algebra over a commutative ring $S$. The Dorroh extension of $R$ by $S$, write $R \oplus_{D} S$, is the ring $R \times S$ with operations $\left(r_{1}, s_{1}\right)+\left(r_{2}, s_{2}\right)=\left(r_{1}+r_{2}, s_{1}+s_{2}\right)$ and $\left(r_{1}, s_{1}\right)\left(r_{2}, s_{2}\right)=$ $\left(r_{1} r_{2}+s_{1} r_{2}+s_{2} r_{1}, s_{1} s_{2}\right)$, where $r_{i} \in R$ and $s_{i} \in S$. 
Example 1.4. According to [17, Example 2.4(3)], let $S$ be the factor ring of the polynomial ring $\mathbb{Z}_{2}\left[t_{1}, t_{2}, \ldots\right]$ with $t_{i}$ 's a set of commuting indeterminates over $\mathbb{Z}_{2}$ modulo the ideal generated by $\left\{t_{i}^{2} \mid i=1,2, \ldots\right\}$ and $T=$ $\left(\begin{array}{cc}N_{0}(S) & S \\ N_{0}(S) & N_{0}(S)\end{array}\right)$, where $\mathbb{Z}_{2}$ is the field of integers modulo 2. Set $R=T \oplus_{D} \mathbb{Z}_{2}$.

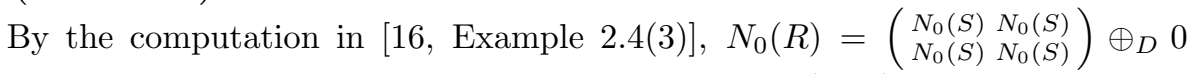
but $N_{0}(R) \varsubsetneqq N_{*}(R)=T \oplus_{D} 0$. Then $R / N_{0}(R) \cong\left(\begin{array}{cc}0 & \mathbb{Z}_{2} \\ 0 & 0\end{array}\right) \oplus_{D} \mathbb{Z}_{2}$. Note that $(a, b) \in R / N_{0}(R)$ is invertible if $b=1$. Suppose $\left(a_{1}, b_{1}\right)\left(a_{2}, b_{2}\right)\left(a_{3}, b_{3}\right)=0$ for $0 \neq\left(a_{i}, b_{i}\right) \in R / N_{0}(R)$ with $i=1,2,3$. Then at least two of $b_{i}$ 's must be zero and so we get $\left(a_{1}, b_{1}\right)\left(a_{3}, b_{3}\right)\left(a_{2}, b_{2}\right)=(c, 0)(d, 0)$ for some $c, d \in\left(\begin{array}{cc}0 & \mathbb{Z}_{2} \\ 0 & 0\end{array}\right)$, entailing $\left(a_{1}, b_{1}\right)\left(a_{3}, b_{3}\right)\left(a_{2}, b_{2}\right)=0$. Thus $R / N_{0}(R)$ is symmetric, but $R$ is not quasi-IFP by the computation in [17, Example 2.4(3)].

The subcondition " $N_{0}(R)=N_{*}(R)$ " $\left(N_{0}(R)=N^{*}(R)\right)$ in the condition (4) $((5))$ in Lemma 1.3 is also not superfluous by Example 1.4.

The $n$ by $n$ upper (lower) triangular matrix rings over a quasi-IFP ring $R$ are also quasi-IFP by [17, Corollary 3.8(1)]. However given any $\operatorname{ring} A, \operatorname{Mat}_{n}(A)$ is not quasi-IFP by Lemma 1.3 when $n \geq 2$. To see that we take the following two examples:

(1) $e_{12}$ and $e_{21}$ are nilpotent but $e_{12}+e_{21}$ is non-nilpotent.

(2) Let $a_{0}=e_{12}, a_{1}=e_{11}-e_{22}$, and $a_{2}=-e_{21}$ and $f(x)=a_{0}+a_{1} x+a_{2} x^{2} \in$ $\operatorname{Mat}_{n}(A)[x]$. Then $f(x)^{2}=0$ but $\sum_{i=0}^{2} \operatorname{Mat}_{n}(A) a_{i} \operatorname{Mat}_{n}(A)$ is non-nilpotent.

In the remainder of this section we study conditions under which various concepts near at the quasi-IFPness coincide.

Semiprime 2-primal rings are reduced and so we obtain the following equivalences by Lemma 1.3 .

Proposition 1.5. Let $R$ be a semiprime ring. Then the following conditions are equivalent:

(1) $R$ is quasi-IFP;

(2) $R$ is IFP;

(3) $R$ is reduced;

(4) $R$ is 2-primal.

When $R$ is a semiprime ring we may conjecture that a ring $R$ is NI if and only if $R$ is reduced, based on Proposition 1.5. However there is a semiprime NI ring but not reduced as we see in [13, Example 1.2].

The index of nilpotency of a nilpotent element $x$ in a ring $R$ is the least positive integer $n$ such that $x^{n}=0$. The index of nilpotency of a subset $I$ of $R$ is the supremum of the indices of nilpotency of all nilpotent elements in $I$. If such a supremum is finite, then $I$ is said to be of bounded index of nilpotency. If $R$ is of bounded index of nilpotency, then $R$ is NI if and only if it is 2-primal by [13, Proposition 1.4]. So we get the following from Proposition 1.5.

Proposition 1.6. Let $R$ be a semiprime ring of bounded index of nilpotency. Then the following conditions are equivalent: 
(1) $R$ is quasi-IFP;

(2) $R$ is IFP;

(3) $R$ is reduced;

(4) $R$ is 2-primal;

(5) $R$ is NI.

The condition "of bounded index of nilpotency" in Proposition 1.6 is not superfluous by [13, Example 1.2] (this ring is semiprime but not of bounded index of nilpotency); while, the condition "semiprime" in Proposition 1.6 is also not superfluous by Example 1.4 (this ring is of bounded index of nilpotency but not semiprime). However " $R$ being quasi-IFP" is not equivalent to them by [17, Example 2.4(3)].

A ring $R$ is called von Neumann regular if for each $a \in R$ there exists $x \in R$ such that $a=$ axa. A ring is called right (resp. left) duo if every right (resp. left) ideal is two-sided. Duo means the two-sided duo. Right or left duo rings are IFP by Lemma 1.1(1), but not conversely as can be seen by [16, Proposition 1.2] and the ring of all matrices of the form $\left(\begin{array}{lll}a & b & c \\ 0 & a & d \\ 0 & 0 & a\end{array}\right)$ over a reduced ring. Von Neumann regular rings need not be quasi-IFP in spite of being semiprime. $\operatorname{Mat}_{n}(R)$ is von Neumann regular by [7, Lemma 1.6] over a von Neumann regular ring $R$, but it is not quasi-IFP by Proposition 1.7 below when $n \geq 2$. In the following we see some conditions under which von Neumann regular rings can be near-IFP.

Proposition 1.7. Let $R$ be a von Neumann regular ring. Then the following conditions are equivalent:

(1) $R$ is right (left) duo;

(2) $R$ is reduced;

(3) $R$ is Abelian;

(4) $R$ is IFP;

(5) $R$ is quasi-IFP;

(6) $R$ is 2-primal;

(7) $R$ is NI.

Proof. Von Neumann regular rings are semiprimitive (hence semiprime) by [7, Corollary 1.2], and so we have the result by [7, Theorem 3.2] and Proposition 1.5 .

A ring $R$ is called strongly regular if for each $a \in R$ there exists $x \in R$ such that $a=a^{2} x$. A ring is strongly regular if and only if it is Abelian and von Neumann regular [7, Theorem 3.5]. From Proposition 1.7 we obtain a similar result to [7, Theorem 3.5].

Corollary 1.8. A ring is strongly regular if and only if it is quasi-IFP and von Neumann regular.

A $\operatorname{ring} R$ is called $\pi$-regular if for each $a \in R$ there exist a positive integer $n=n(a)$, depending on $a$, and $x \in R$ such that $a^{n}=a^{n} x a^{n}$. Von Neumann 
regular rings are clearly $\pi$-regular but the converse need not hold as can be seen by the 2 by 2 upper triangular matrix rings over division rings. From Proposition 1.7, one may conjecture that $\pi$-regular 2-primal (or NI) rings are quasi-IFP. But the following erases the possibility.

Example 1.9. Set $R=T \oplus_{D} \mathbb{Z}_{2}$ be the ring in Example 1.4. Then $R$ is not quasi-IFP. Note that $N_{*}(R)=T \oplus_{D} 0$ and $R / N_{*}(R) \cong \mathbb{Z}_{2}$, getting that $R$ is 2 -primal. Now we will show that $R$ is $\pi$-regular. Take $(t, 0) \in N_{*}(R)$, then $(t, 0) \in J(R)$ and so $(t, 1)=(0,1)-(t, 0)$ is invertible. Consequently each element in $R$ is either nilpotent or invertible, and thus $R$ is $\pi$-regular. Note that $R$ is not von Neumann regular since $J(R)$ is nonzero.

In Example 1.9, let $(n, 1)^{2}=(n, 1)$. Then $n^{2}=n$ and so $n$ must be zero because $n$ is nilpotent; hence all idempotents in $R$ are 0 and 1 , obtaining that $R$ is Abelian. Consequently the ring $R$ in Example 1.8 is Abelian $\pi$-regular that is 2-primal but not quasi-IFP. While, Badawi proved that Abelian $\pi$-regular rings are NI [1, Theorem 3]. So one may ask whether Abelian $\pi$-regular rings may be 2-primal. We also answer that negatively in the following.

Example 1.10. Let $S$ be a division ring and denote by $U_{n}$ the $2^{n}$ by $2^{n}$ upper triangular matrix ring over a $\operatorname{ring} S$, where $n$ is a positive integer. Consider a subring of $U_{n}$

$$
D_{n}=\left\{M \in U_{n} \mid \text { the diagonal entries of } M \text { are equal }\right\} .
$$

Define a map $\sigma: D_{n} \rightarrow D_{n+1}$ by $A \mapsto\left(\begin{array}{cc}A & 0 \\ 0 & A\end{array}\right)$. Then $D_{n}$ can be considered as a subring of $D_{n+1}$ via $\sigma$ (i.e., $A=\sigma(A)$ for $a \in D_{n}$ ). Set $R$ be the direct limit of the direct system $\left(D_{n}, \sigma_{i j}\right)$, where $\sigma_{i j}=\sigma^{j-i}$. Then $R$ is a semiprime ring by $[15$, Theorem 2.2]. Note

$$
N^{*}(R)=N(R)=\{M \in R \mid \text { the diagonal entries of } M \text { are zero }\} .
$$

Let $A \in R$. Then $A \in D_{n}$ for some $n$ and so $A$ is either invertible (when the diagonal of $A$ is nonzero) or nilpotent (when the diagonal of $A$ is zero); hence $R$ is $\pi$-regular. Moreover $R$ is Abelian by [10, Lemma 2]. However $R$ is not 2-primal since $N_{*}(R)=0$ and $N(R) \neq 0$.

Proposition 1.11. Let $R$ be an one-sided Goldie ring or $R$ satisfies ACC on left and right annihilators. Then the following conditions are equivalent:

(1) $R$ is quasi-IFP;

(2) $R$ is 2-primal;

(3) $R$ is NI.

Proof. If $R$ is an one-sided Goldie ring or satisfies ACC on left and right annihilators, then nil ideals are nilpotent by [4, Theorem 1.3.4] and [19]. So NI rings are quasi-IFP by Lemma 1.3 since $N(R)=N_{0}(R)$.

In the following we see another equivalent condition of IFP rings. 
Proposition 1.12. A ring $R$ is IFP if and only if every finitely generated subring of $R$ is IFP.

Proof. It suffices to show the sufficiency. Let the necessity hold and assume on the contrary that $R$ is not IFP. Then there are $a, b, c \in R$ such that $a c=0$ but $a b c \neq 0$. Consider the subring $S$ of $R$ generated by $a, b, c$. Then $S$ is IFP by the necessity and so $a b c=0$, a contradiction. Thus $R$ is IFP.

However this result need not hold for quasi-IFP rings as follows. Due to Huh et al. [11], a ring is called locally finite if every finite subset in it generates a finite semigroup multiplicatively. A ring $R$ is locally finite if and only if each finite subset of $R$ generates a finite subring (not necessarily with identity) if and only if $R / I$ and $I$ are both locally finite for a proper ideal of $R$ [11, Proposition 2.1 and Theorem 2.2].

Proposition 1.13. For a locally finite ring $R$ the following conditions are equivalent:

(1) $R$ is NI;

(2) Every finitely generated subring of $R$ is quasi-IFP;

(3) Every finitely generated subring of $R$ is 2-primal;

(4) Every finitely generated subring of $R$ is $N I$;

(5) For every finitely generated subring $S$ of $R, S / J(S)$ is a finite direct product of finite fields

Proof. Since $R$ is locally finite, every finitely generated subring of $R$ is finite. So we have the equivalences from (2) to (5) with the help of $[17$, Proposition 2.3], noting $N^{*}(S)=N_{*}(S)=J(S)=N_{0}(S)$ for a finite ring $S$. (1) $\Rightarrow(4)$ and $(4) \Rightarrow(1)$ are obtained by [13, Proposition 2.4(2)] and [13, Lemma 2.1] respectively.

The quasi-IFPness and 2-primalness cannot be equivalent to the conditions in Proposition 1.13 by the following.

Example 1.14. We use the construction and computation in [13, Example 1.2]. Let $S$ be a finite field, $n$ be a positive integer and $R_{n}$ be the $2^{n}$ by $2^{n}$ upper triangular matrix ring over $S$. Then each $R_{n}$ is a finite ring, and quasi-IFP by Lemma 1.3. According to [13, Example 1.2], define a map $\sigma: R_{n} \rightarrow R_{n+1}$ by $A \mapsto\left(\begin{array}{cc}A & 0 \\ 0 & A\end{array}\right)$. Then $R_{n}$ can be considered as a subring of $R_{n+1}$ via $\sigma$ (i.e., $A=\sigma(A)$ for $A \in R_{n}$ ). Let $D=\left\{R_{n}, \sigma_{n m}\right\}$ (with $\sigma_{n m}=\sigma^{m-n}$ whenever $n \leq m$ ) be the direct system over $I=\{1,2, \ldots\}$, and $R=\lim _{n} R_{n}$ be the direct limit of $D$. Then $R$ is an NI ring by [13, Proposition 1.1$]$, but not 2 -primal by the computation in [13, Example 1.2]. Considering any finitely generated subring $S$ of $R$, it is clearly a subring of $R_{n}$ for some $n$; hence $S$ is quasi-IFP by Lemma 1.1(4). 


\section{Structure of quasi-IFP rings}

In this section we study various properties of quasi-IFP rings. In [17, Theorem 2.5] we see a characterization of quasi-IFP rings relating to minimal prime ideals. We furthermore find another characterization of quasi-IFP rings via strongly prime ideals as follows. To do that we introduce the following concepts which are essentially due to Shin [23]. Let $R$ be a ring and $P$ be a strongly prime ideal of $R$.

$$
\begin{aligned}
M(P) & =\left\{a \in R \mid a R b \subseteq N_{0}(R) \text { for some } b \in R \backslash P\right\} ; \\
M_{P} & =\left\{a \in R \mid a b \in N_{0}(R) \text { for some } b \in R \backslash P\right\} ; \\
\bar{M}_{P} & =\left\{a \in R \mid a^{m} \in M_{P} \text { for some positive integer } m\right\} .
\end{aligned}
$$

Note that $M(P) \subseteq P, N(R) \subseteq \bar{M}_{P}$, and $M(P) \subseteq M_{P} \subseteq \bar{M}_{P}$. Given a multiplicative monoid $X$ in $R \backslash 0$, if $Q$ is an ideal of $R$ maximal with respect to the property $Q \cap X=\emptyset$, then $Q$ is a strongly prime ideal of $R$ [13, Lemma 2.2].

Theorem 2.1. Given a ring $R$ the following conditions are equivalent:

(1) $R$ is quasi-IFP;

(2) For any minimal strongly prime ideal $P$ of $R$ we have that $M(P)=$ $M_{P}=\bar{M}_{P}=P$, and if $a \in R$ and $a R b \subseteq N_{0}(R)$ for some $b \in R \backslash P$, then $a \in N_{0}(R)$.

Proof. We use Lemma 1.3 freely and apply the proof of [23, Theorem 1.8] to strongly prime ideals and $N_{0}(R)$.

$(1) \Rightarrow(2)$ : Let $P$ be a strongly prime ideal of $R$. We already have $M(P) \subseteq$ $M_{P} \subseteq \bar{M}_{P}$. Let $a \in \bar{M}_{P}$. Then $a^{k} b \in N_{0}(R)$ for some $b \in R \backslash P$ and positive integer $k$. Since $R$ is quasi-IFP, $R / N_{0}(R)$ is reduced and so we have $a R b \subseteq$ $N_{0}(R)$ by $[18$, Section $1(\mathrm{G})]$ and this implies $a \in M(P)$; hence we get $M(P)=$ $M_{P}=\bar{M}_{P}$. Next we will show

$$
M(P)=\bigcap\{Q \mid Q \text { is a strongly prime ideal of } R \text { with } Q \subseteq P\} .
$$

If $Q$ is a strongly prime ideal of $R$ with $Q \subseteq P$, then $M(P) \subseteq M(Q) \subseteq Q$; hence we have $M(P) \subseteq \bigcap\{Q \mid Q$ is a strongly prime ideal of $R$ with $Q \subseteq P\}$.

Conversely, let $a \notin M(P)$. Then the multiplicative subset $S=\left\{a, a^{2}, a^{3}, \ldots\right\}$ of $R$ does not contain any element of $N_{0}(R)$ (if $a^{n} \in N_{0}(R)$, then $a \in N_{0}(R)$ by the reducedness of $\left.R / N_{0}(R)\right)$ because $M(P)=M_{P}=\bar{M}_{P}$. Define

$$
\begin{array}{r}
T=\left\{a^{t_{0}} b_{1} a^{t_{1}} b_{2} \cdots a^{t_{m-1}} b_{m} a^{t_{m}} \notin N_{0}(R) \mid m \in \mathbb{Z}^{+}, b_{i} \in R \backslash P,\right. \\
\left.t_{j} \in \mathbb{Z}^{+} \text {for } j=1,2, \ldots, m-1 \text { and } t_{0}, t_{m} \in\{0\} \cup \mathbb{Z}^{+}\right\},
\end{array}
$$

where $\mathbb{Z}^{+}$is the set of all positive integers. Then $T$ contains both $S$ and $R \backslash P$ (so $1 \in T)$. We claim that $T$ is a multiplicative monoid in $R \backslash 0$. Consider $x, y \in T$. If $x, y \in S$, then $x y \in S \subseteq T$ clearly. If $x=a^{s} \in S, y=a^{t_{0}} b_{1} a^{t_{1}} b_{2} \cdots b_{m} a^{t_{m}} \in$ $T$, then $x y \notin N_{0}(R)$. For, if $x y \in N_{0}(R)$, then $x y=a^{s+t_{0}} b_{1} a^{t_{1}} b_{2} \cdots b_{m} a^{t_{m}} \in$ $N_{0}(R)$. Since $R / N_{0}(R)$ is reduced we have $\left[\left(a^{s+t_{0}+\cdots+t_{m}}\right)\left(b_{1} \cdots b_{m}\right)\right]^{m+1} \in$ 
$N_{0}(R)$ with the help of $[18$, Section $1(\mathrm{G})]$, entailing $\left(a^{s+t_{0}+\cdots+t_{m}}\right)\left(b_{1} \cdots b_{m}\right) \in$ $N_{0}(R)$. But $P$ is prime and so there exist $r_{1}, \ldots, r_{m-1} \in R$ such that $b_{1} r_{1} \ldots$ $b_{m-1} r_{m-1} b_{m} \in R \backslash P$. Letting $k=s+t_{0}+\cdots+t_{m}$ and $b=b_{1} r_{1} \cdots b_{m-1} r_{m-1} b_{m}$, then we have $a^{k} b \in N_{0}(R)$ because $R / N_{0}(R)$ is reduced; hence $a \in \bar{M}_{P}$ and then $a \in M(P)$ since $M(P)=M_{P}=\bar{M}_{P}$. This is a contradiction, concluding $x y \notin N_{0}(R)$ and $x y \in T$. For the cases of $(x, y \in T)$ and $(x \in T, y \in S)$ we can get $x y \notin N_{0}(R)$ (so $x y \in T$ ) by similar computations. Thus $T$ is a multiplicative monoid in $R \backslash 0$. By [13, Lemma 2.2] there exists a strongly prime ideal of $R$, say $J$, that is disjoint from $T$. Then $a \notin J$ and $J \subseteq P$, obtaining $M(P) \supseteq \bigcap\{Q \mid Q$ is a prime ideal of $R$ with $Q \subseteq P\}$. Thus $M(P)=\bigcap\{Q \mid$ $Q$ is a strongly prime ideal of $R$ with $Q \subseteq P\}$. Now suppose that $P$ is any minimal strongly prime ideal of $R$. Then we get $M(P)=P$ from $M(P)=$ $\bigcap\{Q \mid Q$ is a strongly prime ideal of $R$ with $Q \subseteq P\}$. Next assume that $a \in R$ and $a R b \subseteq N_{0}(R)$ for some $b \in R \backslash P$. Note $a R b \subseteq N_{0}(R) \subseteq P$; hence $a \in P$ since $P$ is prime. Since $P$ is arbitrarily taken, we get $a \in N^{*}(R)$. But $R$ is quasi-IFP, and so we have $N_{0}(R)=N^{*}(R)$. Thus $a \in N_{0}(R)$.

$(2) \Rightarrow(1)$ : Suppose that the condition (2) holds. Let $a \in N(R)$ with $a^{m}=0$. Then $a \in \bar{M}_{P}$, and so $a \in M_{P}=M(P)$ for any minimal strongly prime ideal $P$ of $R$. Thus $a R b \subseteq N_{0}(R)$ for some $b \in R \backslash P$ and hence $a \in N_{0}(R)$. Thus $N(R)=N_{0}(R)$ and so $R$ is quasi-IFP by Lemma 1.3 .

We apply Theorem 2.1 to the non-quasi-IFP ring in Example 1.2(2). Let $R$ be the ring in Example 1.2(2). Note that $R(y+I) R$, say $P$, is the unique minimal strongly prime ideal of $R$ with $P=N_{*}(R)=N^{*}(R)=N(R)=\bar{M}_{P}$ since $R / P \cong K[x]$. However $y+I \notin M_{P}$ and thus $R$ is not quasi-IFP by Theorem 2.1.

Huh et al. showed that $R[x]$ need not be IFP when $R$ is an IFP ring $[12$, Example 2]. But $R[X]$ can be quasi-IFP over a quasi-IFP ring $R$ as in the following.

Proposition 2.2. If $R$ is a quasi-IFP ring, then so is $R[X]$.

Proof. Let $R$ be a quasi-IFP ring and $f=a_{0}+\sum_{i=1}^{n} a_{i} P_{i} \in N(R[X])$, where each $P_{i}$ is a finite product of indeterminates in $X$. Then by Lemma 1.3(2), $\sum_{i=0}^{n} R a_{i} R$ is nilpotent and thus

$$
\sum_{i=0}^{n} R[X]\left(R a_{0} R+\sum_{i=1}^{n} R a_{i} R P_{i}\right) R[X]
$$

is also nilpotent. But $R[X] f R[X] \subseteq \sum_{i=0}^{n} R[X]\left(R a_{0} R+\sum_{i=1}^{n} R a_{i} R P_{i}\right) R[X]$ and so $R[X] f R[X]$ is nilpotent; hence $R[X]$ is quasi-IFP by Lemma 1.3 .

Proposition 2.2 is also obtained from [17, Theorem 3.2], but the preceding proof is a simpler one. From Proposition 2.2, it is natural to ask whether the quasi-IFPness is preserved by power series ring extensions. However the answer 
is negative by [17, Example 3.3]. But if a $\operatorname{ring} R$ is 2-primal with nilpotent $N_{0}(R)$, then the power series ring over $R$ is quasi-IFP by [17, Corollary 3.5(1)].

We next obtain a useful method by which given rings are examined to be quasi-IFP. A proper subrings $S$ (possibly without identity) of given a ring can be defined to be quasi-IFP when $S$ satisfies the conditions in Lemma 1.3. The following is equal to [17, Proposition 3.10], but here we take another proof using the properties of $N_{0}(R)$.

Proposition 2.3. Let $R$ be a ring and $I$ be a proper ideal of $R$. If $R / I$ and $I$ are both quasi-IFP rings with $N_{0}(I)$ nilpotent, then $R$ is quasi-IFP.

Proof. We use Lemma 1.3 freely. Since $I$ is quasi-IFP, $N(I)=N_{0}(I)$. We first claim $N_{0}(I)$ is an ideal of $R$ contained in $N_{0}(R)$. Notice $\left\langle N_{0}(I)\right\rangle^{3} \subseteq N_{0}(I)$ by the Andrunakievich Lemma [5, p. 107], where $\left\langle N_{0}(I)\right\rangle$ is the ideal of $R$ generated by $N_{0}(I)$. But since $I / N_{0}(I)$ is nil-semisimple from $N(I)=N_{0}(I)$, we get $\left\langle N_{0}(I)\right\rangle^{3}=N_{0}(I)$, concluding that $N_{0}(I)$ is an ideal of $R$. Next for $a \in N_{0}(I)$, we have $(a I)^{m}=0$ for some positive integer $m$. Then we have $(a R)^{2 m}=0$ from $(a R a R)^{m} \subseteq(a I)^{m}=0$, obtaining $a \in N_{0}(R)$.

Now take $0 \neq d \in N(R)$ with $d^{k}=0$. Since $R / I$ is quasi-IFP, $N(R / I)=$ $N_{0}(R / I)$ and so $(d R)^{\ell} \subseteq I$ for some positive integer $\ell$. Note $(d R)^{\ell} d^{k}=0 \subseteq$ $N_{0}(I)$. Since $k \geq 2,\left((\bar{d} R)^{\ell} d^{k-1}\right)^{3} \subseteq N_{0}(I)$ by the reducedness of $I / N_{0}(I)$, entailing $(d R)^{\ell} d^{k-1} \subseteq N_{0}(I)$. Inductively we get $(d R)^{\ell} d \subseteq N_{0}(I)$. It then follows, from $N_{0}(I)$ being nilpotent, that $(d R)^{(\ell+1) h}=\left((d R)^{\ell} d R\right)^{h}=0$ for some positive integer $h$, concluding $d \in N_{0}(R)$. Thus $R$ is quasi-IFP.

With the help of Proposition 2.3, the upper (lower) triangular matrix rings over quasi-IFP rings are also quasi-IFP. If the ideal $I$ is nilpotent, then we can obtain the following from Proposition 2.3.

Corollary 2.4. Let $R$ be a ring and $I$ be a proper ideal of $R$. If $R / I$ is quasiIFP and $I$ is nilpotent, then $R$ is quasi-IFP.

In Corollary 2.4, consider a weaker condition " $I$ is nil" instead of the condition " $I$ is nilpotent". However there can be counterexamples as we see in the following.

Example 2.5. Let $T$ be a reduced ring, $n$ be a positive integer and $R_{n}$ be the $2^{n}$ by $2^{n}$ upper triangular matrix ring over $T$. Define a map $\sigma: R_{n} \rightarrow R_{n+1}$ by $A \mapsto\left(\begin{array}{cc}A & 0 \\ 0 & A\end{array}\right)$. Then $R_{n}$ can be considered as a subring of $R_{n+1}$ via $\sigma$ (i.e., $A=\sigma(A)$ for $\left.A \in R_{n}\right)$. Let $R$ be the direct limit of the direct system $\left(R_{n}, \sigma_{i j}\right)$, where $\sigma_{i j}=\sigma^{j-i}$. Put

$$
I=\{M \in R \mid \text { each diagonal entry of } M \text { is zero }\} .
$$

Then $I$ is a nil ideal of $R$ such that $R / I$ is reduced (hence quasi-IFP). But $R$ is not 2-primal (hence not quasi-IFP) by the computation in [13, Example 1.2].

$G F\left(p^{n}\right)$ means the Galois field of order $p^{n}$ and $\mathbb{Z}_{n}$ means the ring of integers modulo $n$. Due to Kim et al. [14], a ring $R$ is called strongly right $A B$ if every 
right annihilator of $R$ is bounded, i.e., it contains a nonzero ideal of $R$. IFP rings are strongly right AB by Lemma 1.1(1) and not conversely by [14].

The following construction is due to Xue [25, Example 2]. Let $A\{x, y\}$ be the free algebra generated by $x, y$ over a ring $A$.

Let $B_{1}=G F(2)\{x, y\} /\left(x^{3}, y^{3}, y x, x^{2}-x y, y^{2}-x y\right)$, where $\left(x^{3}, y^{3}, y x, x^{2}-\right.$ $\left.x y, y^{2}-x y\right)$ is the ideal of $G F(2)\{x, y\}$ generated by $x^{3}, y^{3}, y x, x^{2}-x y, y^{2}-x y$.

Let $\mathbb{Z}_{4}=\{\overline{0}, \overline{1}, \overline{2}, \overline{3}\}$ be the ring of integers modulo 4 and

$$
B_{2}=\mathbb{Z}_{4}\{x, y\} /\left(x^{3}, y^{3}, y x, x^{2}-x y, x^{2}-\overline{2}, y^{2}-\overline{2}, \overline{2} x, \overline{2} y\right),
$$

where $\left(x^{3}, y^{3}, y x, x^{2}-x y, x^{2}-\overline{2}, y^{2}-\overline{2}, \overline{2} x, \overline{2} y\right)$ is the ideal of $\mathbb{Z}_{4}\{x, y\}$ generated by $x^{3}, y^{3}, y x, x^{2}-x y, x^{2}-\overline{2}, y^{2}-\overline{2}, \overline{2} x, \overline{2} y$.

Let $B_{3}$ be the ring of all matrices of the form $\left(\begin{array}{ll}a & b \\ 0 & a^{2}\end{array}\right)$ over $G F\left(2^{2}\right)$.

Xue showed that each $B_{i}$ is a noncommutative duo ring of order 16 [24, Proposition 3]. Note that $C h\left(B_{1}\right)=2=C h\left(B_{3}\right), C h\left(B_{2}\right)=4$ and $\left|J\left(B_{1}\right)\right|=8$, $\left|J\left(B_{3}\right)\right|=4$. Thus $B_{1} \nsucceq B_{2}, B_{1} \nsucceq B_{3}$ and $B_{2} \nsucceq B_{3}$. Xue also showed that any minimal noncommutative duo ring is isomorphic to $B_{i}$ for some $i=1,2,3$ by [25, Theorem 3]. The term "minimal" means "having smallest cardinality".

Theorem 2.6. (1) Every minimal noncommutative quasi-IFP ring is isomorphic to $U_{2}(G F(2))$.

(2) Every minimal noncommutative IFP ring $R$ is isomorphic to $B_{k}$ for some $k \in\{1,2,3,4\}$, where $B_{i}$ 's are the rings above for $i=1,2,3$ and

$$
B_{4}=\left\{\left(\begin{array}{ccc}
a & b & c \\
0 & a & d \\
0 & 0 & a
\end{array}\right) \mid a, b, c, d \in G F(2)\right\} .
$$

Proof. Eldridge proved that if $R$ is a finite noncommutative ring of order $p^{3}$ for a positive prime $p$, then $R$ is isomorphic to $U_{2}(G F(p))$ [6, Proposition], and that if $R$ is a ring of finite order whose factorization is cube free, then $R$ is commutative [6, Theorem]. Thus every minimal noncommutative ring is isomorphic to $U_{2}(G F(2))$.

(1) Note that $U_{2}(G F(2))$ is quasi-IFP by Lemma 1.3, and so we conclude that $U_{2}(G F(2))$ is the minimal noncommutative quasi-IFP ring up to isomorphism.

(2) Recall that one-sided duo rings are IFP and IFP rings are strongly right AB. The result (1) is non-available to IFP rings since IFP rings are Abelian. But Kim et al. proved that $B_{4}$ is a minimal noncommutative strongly right $\mathrm{AB}$ ring [14, Theorem 2.6(1)]; hence the order of $R$ must be 16 because $B_{4}$ is also IFP by [16, Proposition 1.2].

Xue showed that each $B_{i}(i=1,2,3)$ is a noncommutative duo (hence IFP) ring of order 16 [24, Proposition 3]. Based on this result and [17, Theorem 2.6(1)], every minimal noncommutative IFP $\operatorname{ring} R$ is isomorphic to $B_{k}$ for some $k \in\{1,2,3,4\}$ up to isomorphism. 


\section{References}

[1] A. Badawi, On abelian $\pi$-regular rings, Comm. Algebra 25 (1997), no. 4, 1009-1021.

[2] H. E. Bell, Near-rings in which each element is a power of itself, Bull. Austral. Math. Soc. 2 (1970), 363-368.

[3] G. F. Birkenmeier, H. E. Heatherly, and E. K. Lee, Completely prime ideals and associated radicals, Ring theory (Granville, OH, 1992), 102-129, World Sci. Publ., River Edge, NJ, 1993.

[4] A. W. Chatters and C. R. Hajarnavis, Rings with Chain Conditions, Research Notes in Mathematics, 44. Pitman (Advanced Publishing Program), Boston, Mass.-London, 1980.

[5] N. J. Divinsky, Rings and Radicals, Mathematical Expositions No. 14 University of Toronto Press, Toronto, Ont. 1965.

[6] D. B. Erickson, Orders for finite noncommutative rings, Amer. Math. Monthly 73 (1966), 376-377.

[7] K. R. Goodearl, von Neumann Regular Rings, Monographs and Studies in Mathematics, 4. Pitman (Advanced Publishing Program), Boston, Mass.-London, 1979.

[8] K.-Y. Ham, Y. C. Jeon, J. Kang, N. K. Kim, W. Lee, Y. Lee, S. J. Ryu, and H.-H. Yang, IFP Rings and Near-IFP Rings, J. Korean Math. Soc. 45 (2008), no. 3, 727-740.

[9] C. Y. Hong and T. K. Kwak, On minimal strongly prime ideals, Comm. Algebra 28 (2000), no. 10, 4867-4878.

[10] C. Huh, H. K. Kim, and Y. Lee, p.p. rings and generalized p.p. rings, J. Pure Appl. Algebra 167 (2002), no. 1, 37-52.

[11] C. Huh, N. K. Kim, and Y. Lee, Examples of strongly $\pi$-regular rings, J. Pure Appl. Algebra 189 (2004), no. 1-3, 195-210.

[12] C. Huh, Y. Lee, and A. Smoktunowicz, Armendariz rings and semicommutative rings, Comm. Algebra 30 (2002), no. 2, 751-761.

[13] S. U. Hwang, Y. C. Jeon, and Y. Lee, Structure and topological conditions of NI rings, J. Algebra 302 (2006), no. 1, 186-199.

[14] S. U. Hwang, N. K. Kim, and Y. Lee, On rings whose right annihilators are bounded, Glasgow Math. J., To appear.

[15] Y. C. Jeon, H. K. Kim, and J. S. Yoon, On weak Armendariz rings, Bull. Korean Math. Soc. 46 (2009), no. 1, 135-146.

[16] N. K. Kim and Y. Lee, Extensions of reversible rings, J. Pure Appl. Algebra 185 (2003), no. 1-3, 207-223.

[17] N. K. Kim, Y. Lee, and S. J. Ryu, An ascending chain condition on Wedderburn radicals, Comm. Algebra 34 (2006), no. 1, 37-50.

[18] J. Lambek, On the representation of modules by sheaves of factor modules, Canad. Math. Bull. 14 (1971), 359-368.

[19] C. Lanski, Nil subrings of Goldie rings are nilpotent, Canad. J. Math. 21 (1969), 904907.

[20] G. Marks, On 2-primal Ore extensions, Comm. Algebra 29 (2001), no. 5, 2113-2123.

[21] L. Motais de Narbonne, Anneaux semi-commutatifs et unisériels; anneaux dont les idéaux principaux sont idempotents, Proceedings of the 106th National Congress of Learned Societies (Perpignan, 1981), 71-73, Bib. Nat., Paris, 1982.

[22] L. H. Rowen, Ring Theory, Academic Press, Inc., Boston, MA, 1991.

[23] G. Shin, Prime ideals and sheaf representation of a pseudo symmetric ring, Trans. Amer. Math. Soc. 184 (1973), 43-60.

[24] W. Xue, On strongly right bounded finite rings, Bull. Austral. Math. Soc. 44 (1991), no. 3, 353-355.

[25] - Structure of minimal noncommutative duo rings and minimal strongly bounded nonduo rings, Comm. Algebra 20 (1992), no. 9, 2777-2788. 
Hong Kee Kim

Department of Mathematics

Gyeongsang National University

JiNJU 660-701, KorEA

E-mail address: hkkim@gsnu.ac.kr

Nam Kyun Kim

Division of General Education

Hanbat National University

DAeJeOn 305-719, Korea

E-mail address: nkkim@hanbat.ac.kr

Mun Seob Jeong

Department of Mathematics

Busan National University

Busan 609-735, Korea

E-mail address: moonsta-r2000@hanmail.net

YANG LEE

Department of Mathematics Education

Busan National University

Busan 609-735, Korea

E-mail address: ylee@pusan.ac.kr

Sung Ju Ryu

Department of Mathematics

Busan National University

Busan 609-735, Korea

E-mail address: sung1530@dreamwiz.com

Dong Eun Yeo

Department of Mathematics

Busan National University

Busan 609-735, KoREA

E-mail address: dongeunyeo@pusan.ac.kr 